\title{
Sustainable inflammation transforms hepatic cells by causing oxidative stress injury and potential epithelial-mesenchymal transition
}

\author{
KUN LU ${ }^{1,2^{*}}$, GUOYAN LIU ${ }^{2 *}$, LING YANG $^{1 *}$, FAN LIU $^{1}$, LIBIN GAO $^{1}$, JINGXIAN SHI $^{1}$, \\ XIAOLING DENG ${ }^{1}$, QIFU LI ${ }^{1}$, DONGHUI XU ${ }^{3}$ and SONGLIN SHI ${ }^{1}$
}

\author{
${ }^{1}$ Department of Basic Medicine, Medical College of Xiamen University/Cancer Research Center of Xiamen University, \\ Xiamen, Fujian 361102; ${ }^{2}$ Zhongshan Hospital, Medical College of Xiamen University, Xiamen, Fujian 361004; \\ ${ }^{3}$ Department of Hepatic Biliary Pancreatic Vascular Surgery, The First Affiliated Hospital \\ of Xiamen University, Xiamen, Fujian 361003, P.R. China
}

Received April 2, 2016; Accepted May 27, 2016

DOI: 10.3892/ijo.2016.3580

\begin{abstract}
The inflammatory microenvironment promotes tumorigenesis. However, the mechanism through which inflammation transforms hepatic cells in precancerous lesions remains unclear. Hepatic cells undergo significant changes in metabolism before carcinogenesis, but the specific alterations in gene expression and cellular functions in response to precancerous inflammation have not been elucidated. In this study, a hepatitis-hepatoma mouse model was successfully established. Label-free quantitative (LFQ) proteomics coupled with bioinformatics analysis was then performed to identify differentially expressed proteins and their functions in hepatic cells with precancerous inflammation. We found that different chemical treatments induced several common changes in the model. Hepatic cells underwent serious oxidative stress injury. Canonical pathway analysis using IPA revealed the activation of signaling pathways, such as integrin signaling, signaling by Rho family GTPases, IL-8 signaling, and ILK signaling, as well as the inhibition of RhoGDI signaling. Analysis of the KEGG pathway indicated alteration in the pathways for focal adhesion and regulation of actin cytoskeleton. Results from western blot analysis demonstrated the upregulation of proteins, including p-STAT3, TWIST, SNAIL, Vimentin, and MMP-9, which are involved in epithelial-mesenchymal transition (EMT). These results indicated that hepatic cells
\end{abstract}

Correspondence to: Dr Songlin Shi, Department of Basic Medicine, Medical College of Xiamen University/Cancer Research Center of Xiamen University, Xiang'an South Road, Xiamen, Fujian 361102, P.R. China

E-mail: shisonglin@xmu.edu.cn

${ }^{*}$ Contributed equally

Key words: inflammation, hepatic cells, label-free proteomic analysis, epithelial-mesenchymal transition, integrin signaling were likely to undergo EMT. Interestingly, the expression of E-cadherin was upregulated, but this observation must be further investigated. In conclusion, the results revealed that notable functional and pathway changes occurred during the precancerous inflammation stage in the liver. Our study contributes to understanding of the roles of inflammation in tumorigenesis and provides a molecular basis for further studies on the tumorigenesis of hepatocellular carcinoma.

\section{Introduction}

Tumor microenvironments have become hotspots in cancer research; of these environments, the inflammatory microenvironment plays a decisive role in tumorigenesis. The accumulated compelling evidence over the last decade indicate the role for inflammation in tumorigenesis, which has attracted increased research attention. Collectively, 15-20\% of all cancer deaths are linked to underlying infections and inflammation $(1,2)$. In particular, hepatocellular carcinoma (HCC), with a high percentage of $>90 \%$, develops because of chronic liver damage and inflammation $(3,4)$. Sustainable inflammation causes the overproduction of various cytokines, growth factors, and cytotoxic media. Cytotoxic media, such as reactive oxygen species (ROS), nitrogen oxygen species (NOS), and metalloproteases, can induce oxidative DNA damage, DNA methylation, and hepatocyte injury. Moreover, the compensatory hepatocyte regeneration triggered by cytokines and growth factors enhances the accumulation of genomic damage, thereby accelerating hepatocarcinogenesis (4-6). The observation that the inflammatory microenvironment promotes tumorigenesis is supported by considerable evidence. However, the mechanism through which the inflammatory microenvironment alters cellular gene expression and phenotype to promote tumorigenesis has not been elucidated.

Diethylnitrosamine (DEN) is widely used as a potent hepatocarcinogenic initiator in animal models of carcinogenesis; this compound induces DNA adduct formation, resulting in DNA mutation $(7,8)$. To explore the relationship between hepatitis and liver cancer development, we performed 
intraperitoneal injection of DEN on C57/BL mouse to induce in vivo hepatocellular tumorigenesis. Parallel experiments with a combination of pyrrolidine dithiocarbamate (PDTC) or lipopolysaccharide (LPS) with DEN were performed to mimic an anti-inflammation or pro-inflammation situation, respectively. In our previous study, we explored the dynamic metabolic changes during hepatitis and liver cirrhosis; the inflammatory environment induces remarkable changes in carbohydrate and lipid metabolism, and D-glucose and D-mannitol can be used as potential early diagnostic biomarkers of HCC (9). In this study, we analyzed the influence of the inflammatory environment on hepatocellular gene expression and functions through LFQ proteomic technology coupled with bioinformatics analysis. The present study aimed to reveal possible regulatory mechanisms of hepatocellular pathological changes during the precancerous inflammation stage.

\section{Materials and methods}

Establishment of hepatitis mouse model. A mouse model of hepatitis was established using previously described methods (9). All chemical compounds used for animal treatment were purchased from Sigma (USA). These compounds included DEN, phenobarbital (PB), PDTC, and LPS. PB was used as tumor promoter to increase the occurrence of cancer. C57BL/6 mice (males, six weeks of age) were obtained from the Xiamen University Laboratory Animal Center. C57BL/6 mice were randomly assigned to four groups and intraperitoneally injected once a week with the following: PBS (control group, $n=12$ ); DEN (DEN group, $\mathrm{n}=24$ ); DEN and PDTC (DEN+PDTC group, $\mathrm{n}=24$ ); or DEN and LPS (DEN+LPS, $\mathrm{n}=24$ ) for up to 24 weeks. The concentrations of the administered chemicals were based on the body weight of the mice: DEN, $80 \mathrm{mg} / \mathrm{kg}$; PDTC, $50 \mathrm{mg} / \mathrm{kg}$; and LPS, $1.25 \mathrm{mg} / \mathrm{kg}$. PB $(0.05 \%)$ was added to the drinking water on the 10th week since the first DEN injection to enhance DEN-induced tumor formation. After 12 weeks of treatment, four mice from each treatment group and two mice from the control group were sacrificed per month to collect liver samples. All animal handling procedures were approved by the Institutional Review Board of Xiamen University.

Hematoxylin and eosin staining. Liver tissues from mice were fixed in $4 \%$ paraformaldehyde for $12-24 \mathrm{~h}$ and embedded in paraffin. Subsequently, $5-\mu \mathrm{m}$ sections were prepared with a microtome, placed on clean polylysine-coated slides, and then heated at $60^{\circ} \mathrm{C}$ for $2 \mathrm{~h}$. After deparaffinization and rehydration, the sections were stained by hematoxylin and eosin (H\&E) according to standard protocols.

Western blot analysis. Liver tissues were homogenized and lysed in RIPA buffer (50 mM Tris-HCl, pH 8.0; $150 \mathrm{mM}$ sodium chloride; $1.0 \%$ Triton X-100; $0.5 \%$ SDS; $0.5 \%$ sodium deoxycholate; $1 \mathrm{mM}$ PMSF; $1 \mathrm{X}$ protease inhibitor cocktail, Roche) at $4^{\circ} \mathrm{C}$ for $30 \mathrm{~min}$. After sonication in an ice bath, the lysates were clarified by centrifugation at $4^{\circ} \mathrm{C}$ and $12,000 \mathrm{x} \mathrm{g}$ for $15 \mathrm{~min}$. Protein concentration was determined by Bradford assay. The proteins were resolved by SDS-PAGE, transferred to a PVDF membrane, and immunoblotted with specific primary and secondary antibodies. The signals were detected by ECL.
Label-free quantitative proteomics analysis. For sample preparation, $100 \mathrm{mg}$ of liver tissues were homogenized and lysed in lysis buffer containing $8 \mathrm{M}$ urea, $50 \mathrm{mM}$ Tris-base (pH 8.0), and $1 \mathrm{X}$ protease inhibitor cocktail (Roche) in an ice bath. After sonication, the lysates were clarified by centrifugation at $4^{\circ} \mathrm{C}$ and $12,000 \times \mathrm{g}$ for $30 \mathrm{~min}$ and then purified via acetone precipitation. The samples were redissolved in urea lysis buffer. Protein concentration was determined by Bradford assay. After reduction in $30 \mathrm{mM}$ DTT and alkylation in $50 \mathrm{mM}$ iodoacetamide (IAA), the proteins were digested by incubation with trypsin (1:50, enzyme to protein) at $37^{\circ} \mathrm{C}$ for $16 \mathrm{~h}$. The samples were desalted by passing them through a SepPak C18 column (Waters). The peptides were analyzed by LC-MS/MS (AB Sciex TripleTOF 5600+). The MS data were analyzed with Maxquant software.

Bioinformatics analysis. Bioinformatics analysis with ingenuity pathway analysis (IPA; Ingenuity ${ }^{\circledR}$ Systems, www. ingenuity.com) included tox list analysis, protein-protein interacting network analysis and canonical pathway analysis. The entire protein list containing the protein accession number (UniprotKB) and the corresponding fold changes was uploaded as an Excel spreadsheet. The protein accession numbers were mapped to their corresponding genes in the Ingenuity Pathways Knowledge Base. The gene list was used for bioinformatics analysis. The cut-off for the fold-change was set to 2.0. KEGG pathway enrichment by DAVID was performed according to the instructions for the database. Given that the database did not identify fold-change data, we only used the differentially expressed proteins for the analysis.

\section{Results}

Pathological changes in mouse liver during the precancerous inflammation stage. To identify pathological changes in mouse liver, we subjected the paraffin sections of mouse liver tissues to H\&E staining. As shown in Fig. 1A (9), the hepatic lobule in normal livers (control group) contained an intact structure, which was surrounded by cords of liver cells that radiated in all directions. Among the liver tissues derived from the treatment groups (DEN, DEN+PDTC, and DEN+LPS groups), a large number of infiltrated inflammatory cells appeared and partially formed focal inflammation.

Identification of differentially expressed proteins during the precancerous inflammation stage was by LFQ proteomic analysis. To compare differences in protein expression between normal and inflammatory liver tissues, we obtained the protein expression profiles of different groups by LFQ proteomic analysis. A total of 2,666 proteins were identified and quantified. To estimate the quantitative capacity of our data set, we first applied box-plot analysis in comparing the average LFQ intensity of the four groups. As shown in Fig. 1B, the average LFQ intensity was similar across all groups; this finding indicated the unbiased result of our data set. To discern the differences in global protein expression across the four groups, the LFQ intensity of differentially expressed proteins were subjected to hierarchical clustering analysis (HCA) by Cluster 3.0. The result was visualized as a heat map (Fig. 1C) with Treeview. The expression profiles of the three treatment groups significantly differed from the control group. 
A

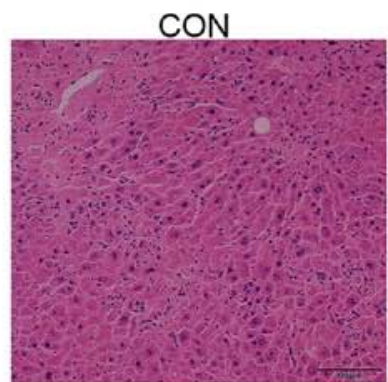

DEN+LPS
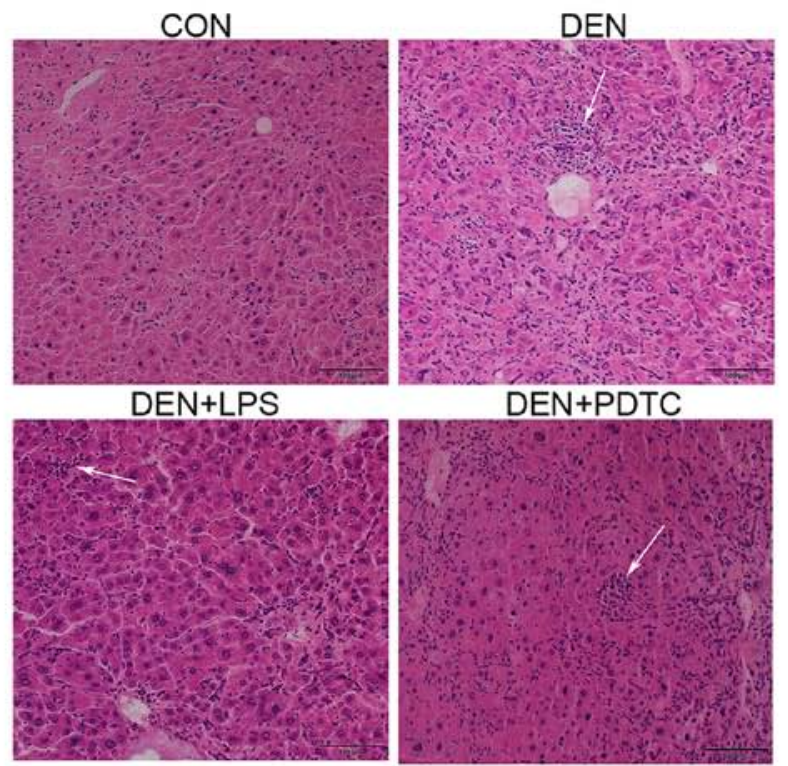

DEN+PDTC

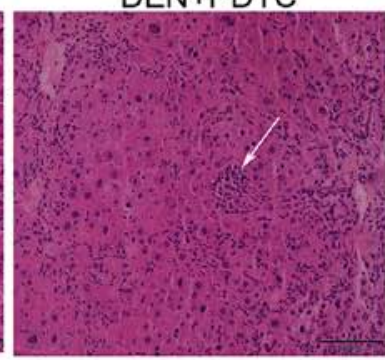

D

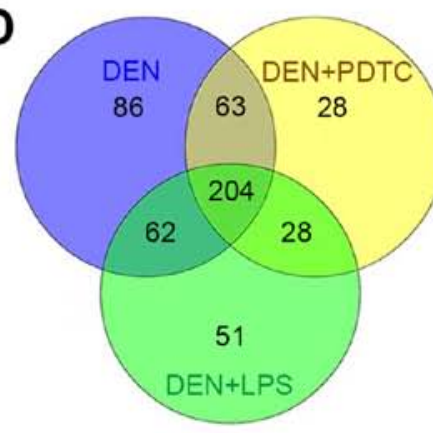

E

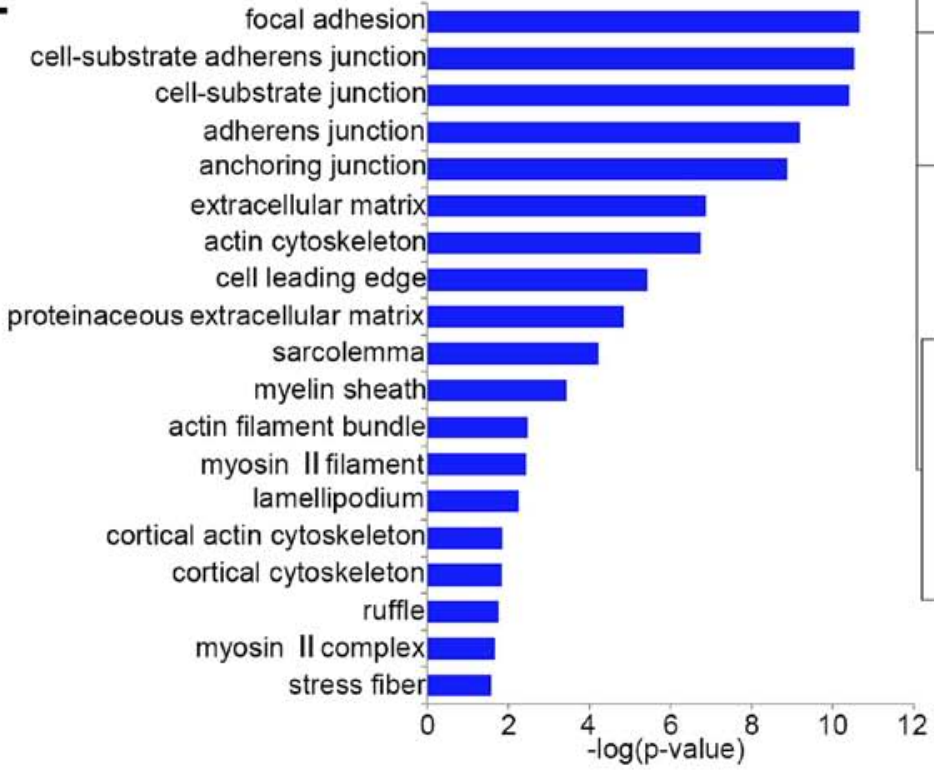

B

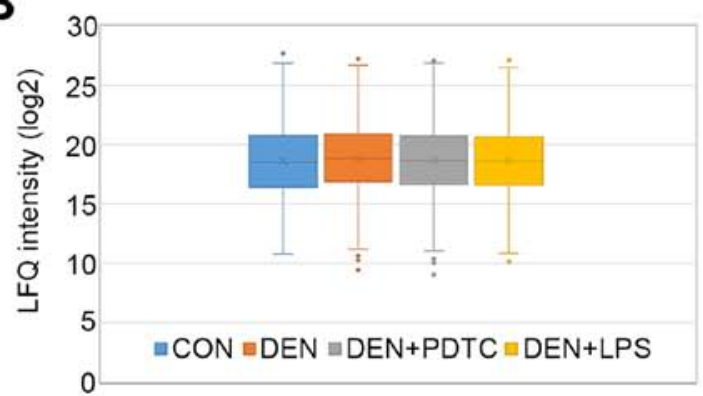

C

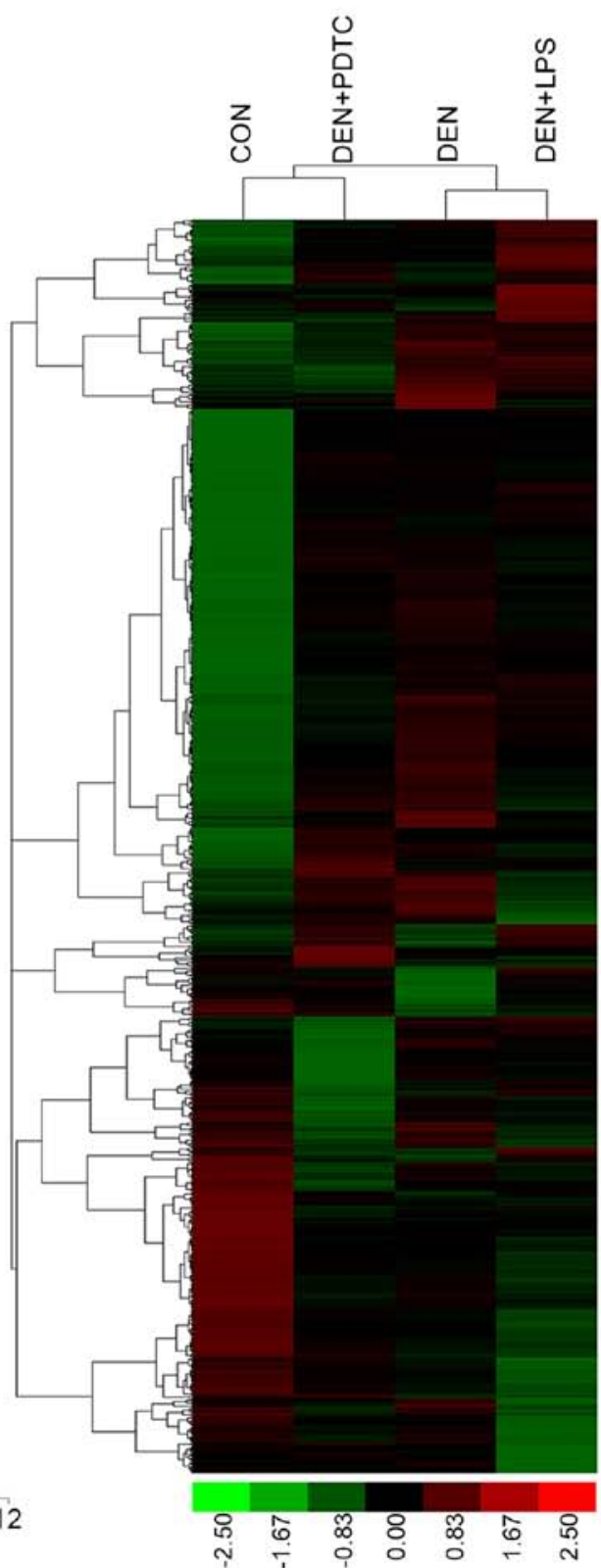

Figure 1. Pathological changes and protein expression profiles of mouse livers demonstrated common inflammation under different treatments. (A) H\&E staining indicated infiltration of inflammatory cells (white arrows) in all three treatment groups (9). Scale bar, $100 \mu \mathrm{m}$. (B) Box plots of protein LFQ intensity $(\log 2)$. The average LFQ intensity was the same across all groups. (C) HCA with Cluster3.0. Data were treated by log transformation, median analysis, and normalization. The following cluster parameters were used: similarity metric, correlation (uncentered); clustering method, complete linkage. (D) Venn diagram showing the distribution of differentially expressed proteins among the three groups. (E) Cellular component enrichment analysis of 204 common upregulated proteins.

A Venn diagram (Fig. 1D) was applied to show the distribution of differentially expressed proteins in the DEN, DEN+PDTC, and DEN+LPS groups. A total of 204 upregu- lated proteins and 62 downregulated proteins were shared by the three treatment groups. Cellular component enrichment toward 204 upregulated proteins was performed with the 


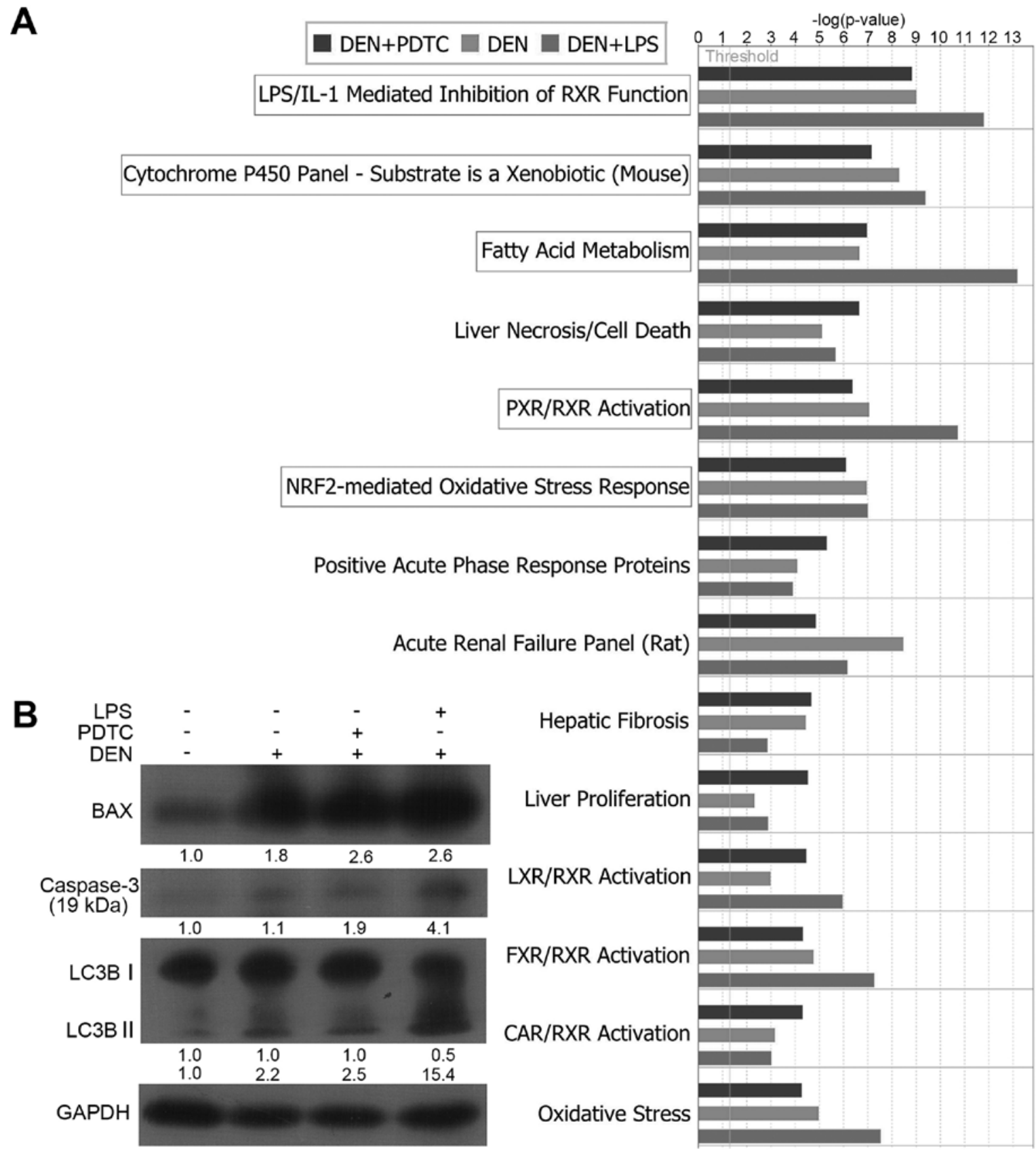

Figure 2. Hepatic cells suffered serious oxidative stress-induced damage. (A) Selected Tox lists associated with our data set analyzed by IPA. The top five Tox lists associated with all three treatment groups are labeled by boxes. (B) Western blot analysis of apoptosis- or autophagy-related proteins. The number below the band indicated protein levels relative to GAPDH. Band intensity was calculated with Quantity One (version 4.6.2, Bio-Rad) and normalized to control group (the first lane on the left).

Gene Ontology database (http://geneontology.org/; powered by PANTHER). These proteins were mainly associated with cell adhesion, cell junctions, and cytoskeleton (Fig. 1E).

Tox list analysis by IPA revealed oxidative stress-induced hepatocellular injury and death during the precancerous inflammation stage. IPA furnishes the analysis of Tox lists to correlate experimental data with clinical pathology endpoints. In IPA, a p-value is calculated using right-tailed Fischer's exact test; this value is always used to measure the likelihood that the association between a set of focus genes in our data and a given term is due to random chance. Low p-values indicate that the association is less likely random but is significant. We selected the threshold of $\mathrm{p}<0.05(-\log \mathrm{p}>1.3$, as indicated by a line in the figures) as the statistically significant level (the same was used in the following canonical pathway analysis). Our data set showed a significant association with the Tox lists of LPS/IL-1-mediated inhibition of RXR function, cytochrome P450 panel-substrate nature as xenobiotic (mouse), fatty acid metabolism, PXR/RXR activation, and NRF2-mediated oxidative stress response (Fig. 2A). All these pathological endpoints were related to oxidative stress.

The pathological endpoint of liver necrosis/cell death $(-\log p>5)$ indicated that hepatic cells might experience a certain 
Table I. Characterization of protein interacting networks.

\begin{tabular}{lccl}
\hline Group & Score & $\begin{array}{c}\text { Focus molecule } \\
\text { number }\end{array}$ & \\
\hline DEN & 54 & 32 & Top diseases and functions \\
DEN & 44 & 28 & Lipid metabolism, nucleic acid metabolism, small molecule biochemistry \\
DEN+PDTC & 64 & 35 & Cellular assembly and organization, DNA replication, recombination, and repair, \\
& & 28 & cardiac arrythmia \\
DEN+PDTC & 44 & 30 & Cipid metabolism, nucleic acid metabolism, small molecule biochemistry \\
DEN+LPS & 48 & 31 & Cardiac stenosis, cardiovascular disease, organismal injury and abnormalities \\
DEN+LPS & 42 & &
\end{tabular}

Molecule number in each network was limited to 35 . Focus molecule number indicated the number of differentially expressed proteins in each network.

Table II. The common activated or inhibited signaling pathways in three treatment groups indicated by IPA.

\begin{tabular}{|c|c|c|c|c|c|c|}
\hline \multirow[b]{2}{*}{ Ingenuity canonical pathways } & \multicolumn{2}{|c|}{ DEN } & \multicolumn{2}{|c|}{$\mathrm{DEN}+\mathrm{PDTC}$} & \multicolumn{2}{|c|}{ DEN+LPS } \\
\hline & State & Z-score & State & Z-score & State & Z-score \\
\hline Integrin signaling & Activated & 2.828 & Activated & 3 & Activated & 2.496 \\
\hline Signaling by Rho family GTPases & Activated & 2.5 & Activated & 2.5 & Activated & 3.3 \\
\hline IL-8 signaling & Activated & 2.496 & Activated & 2 & Activated & 2.982 \\
\hline ILK signaling & Activated & 2.496 & Activated & 2.138 & Activated & 2.84 \\
\hline RhoGDI signaling & Inhibited & -2.121 & Inhibited & -2.53 & Inhibited & -2.309 \\
\hline
\end{tabular}

Z-score $\geq 2$ or $\leq-2$ was selected as an indication of activated or inhibited state.

degree of damage and death. We detected the expression levels of some proteins associated with cell apoptosis and autophagy. As shown in Fig. 2B, the levels of the activated fragment of Caspase-3, BAX, and the small fragment of LC3B significantly increased in the DEN, DEN+PDTC, and DEN+LPS groups.

Identification of significant protein functional groups by establishing protein-interacting networks. The functions of proteins highly rely on their interactions. By establishing protein-interacting networks, thousands of proteins can be assigned to different functional groups, which facilitate the analysis of regulatory events in the proteomics data. We used the IPA software to map the differentially expressed proteins into corresponding protein-protein interaction networks. The networks of corresponding molecules were algorithmically generated based on their connectivity and assigned a score. This score took into account the number of focus molecules derived from our input dataset and the size of the network to approximate how relevant this network was to our original dataset. However, the score might not be an indication of the quality or significance of the network. The intensity of the color in the network represented the degree of upregulation (red) or downregulation (green) of molecules in our dataset. The uncolored genes did not belong to our dataset, but were integrated into the network because of their relevance as indicated by IPA.
A total of 25 protein-interacting networks were acquired from each treatment group. The top two networks of each group with the highest scores are shown in Fig. 3, and the involved functions are listed in Table I. Centered on HNF4A, actin, GRB2, LMNA, EGFT, and ICAM1, these networks were involved in cellular assembly and organization, DNA replication, recombination, and repair, developmental disorders, hereditary disorders, the cell cycle, lipid metabolism, nucleic acid metabolism, and organismal injury and abnormalities.

Pathway analysis by IPA and DAVID revealed that sustainable inflammation enhanced the EMT ability in hepatic cells. The signaling pathways of the differentially expressed proteins can explain how the inflammatory microenvironment transforms hepatic cells. Consequently, we performed canonical pathway analysis with the IPA software. Additional to the p-values calculated by the Fischer's exact test, IPA gave a Z-score to indicate if the pathway was in an activated state $(Z>0)$ or inhibited state $(\mathrm{Z}<0)$. The $\mathrm{Z}$-score was calculated according to the expression level (up or down) of a corresponding molecule in the data set and the expected expression value (up or down) in the pathway. We selected $Z \geq 2$ and $Z \leq-2$ as indicators of the activated and inhibited states, respectively. As shown in Table II and Fig. 4A, integrin signaling, signaling by Rho family GTPases, IL-8 signaling, and ILK signaling were the common activated pathways among the DEN, DEN+PDTC, 
A

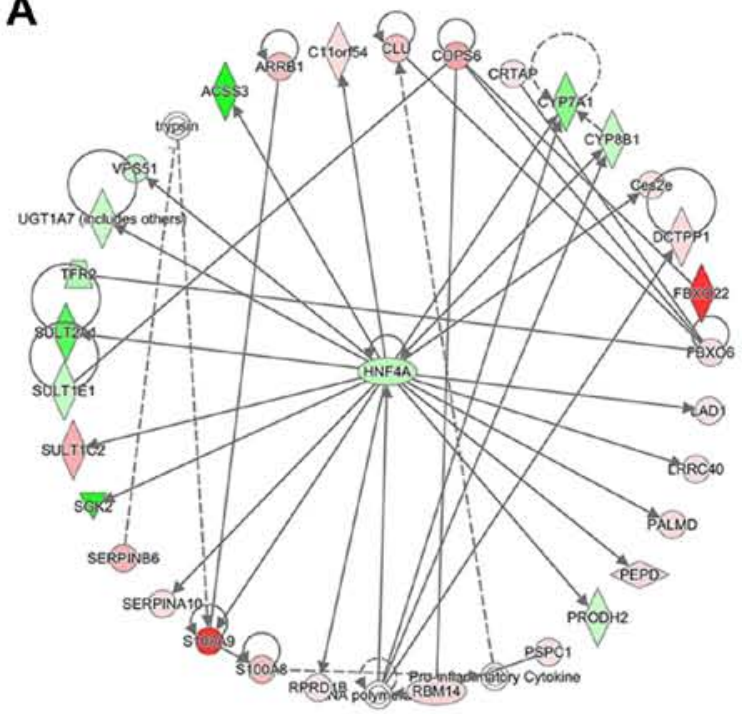

C

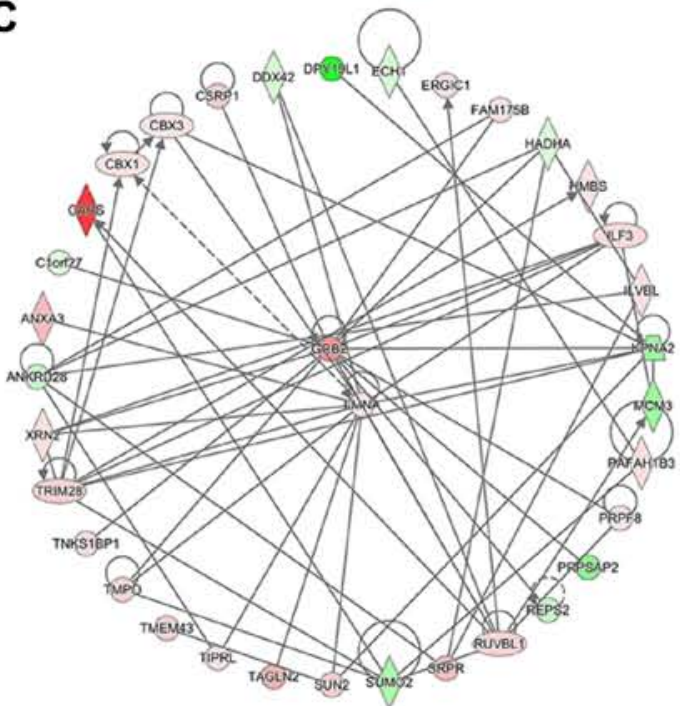

$\mathbf{E}$

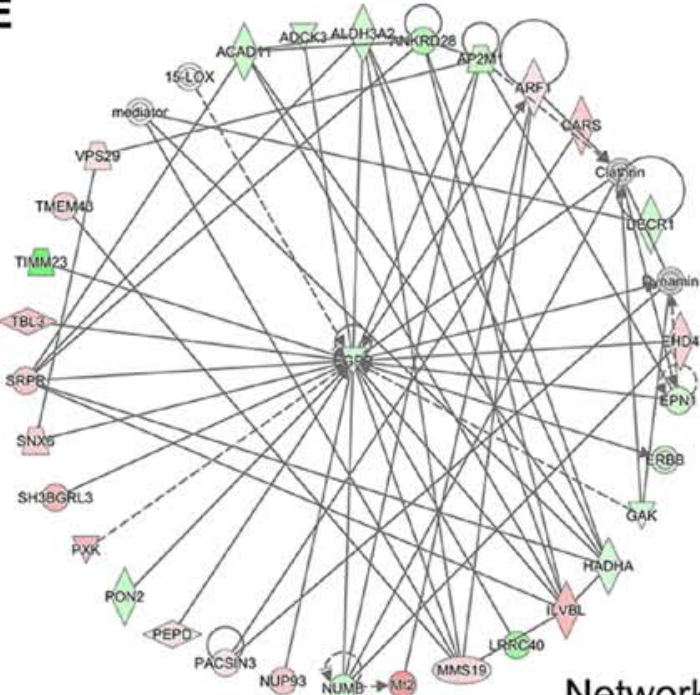

B

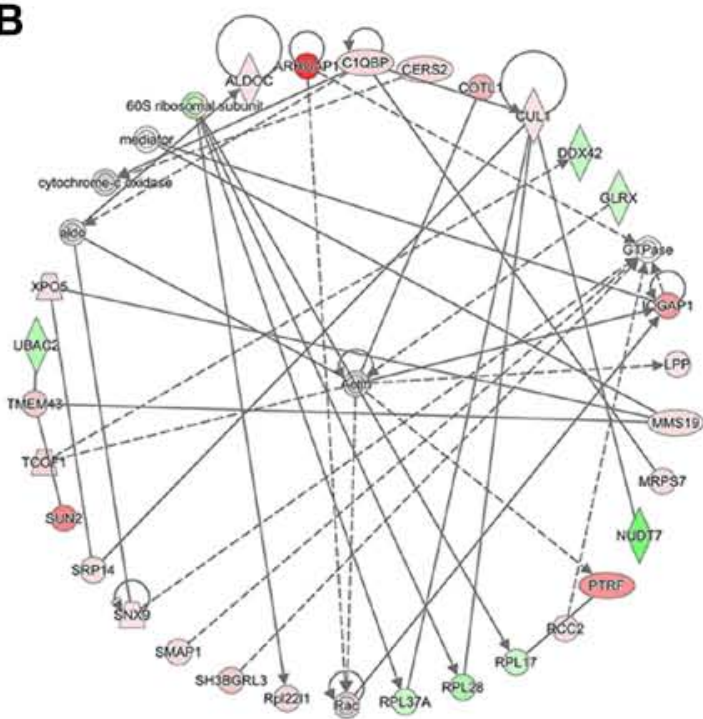

D

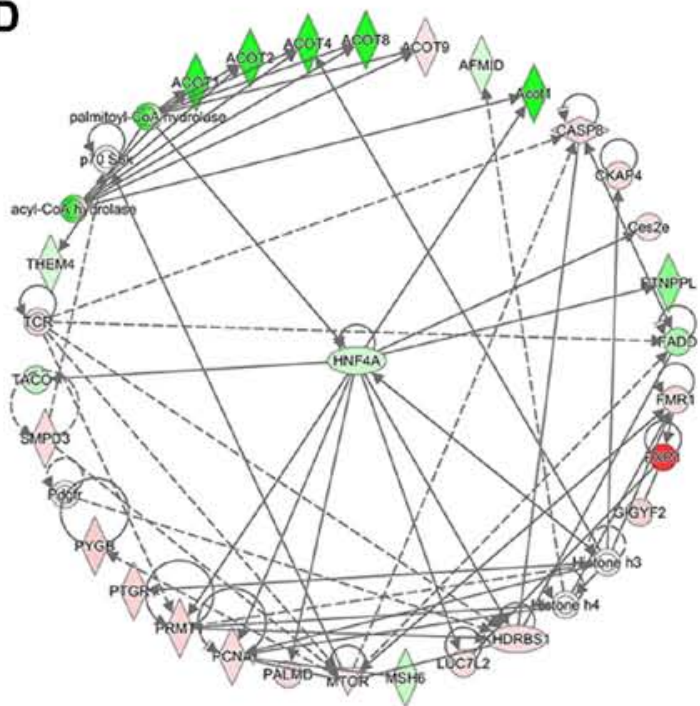

$\mathbf{F}$

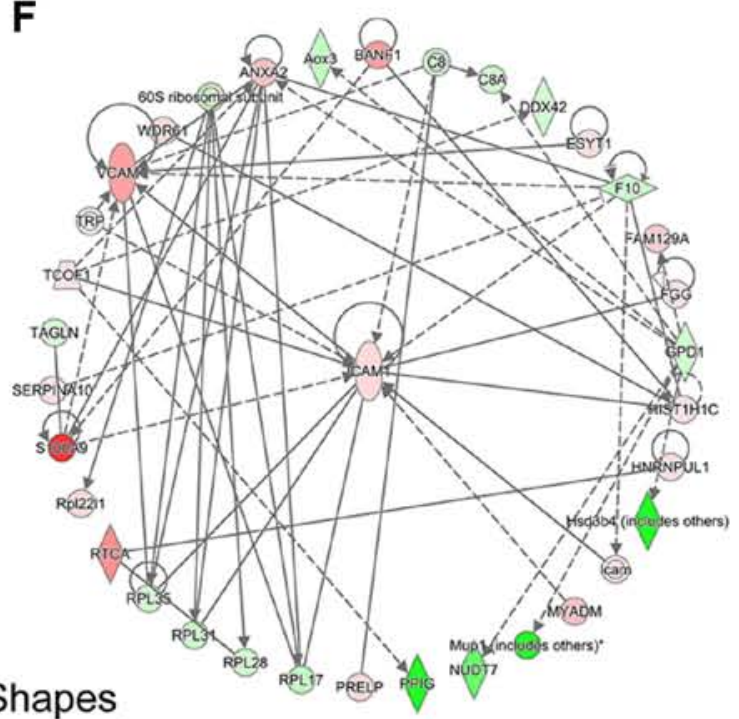

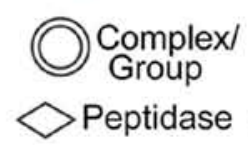

Network Shapes

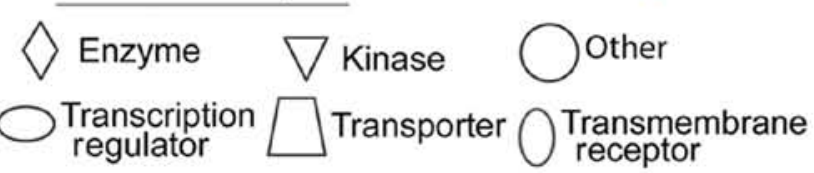

Figure 3. Six protein-interacting networks with top scores obtained from IPA. (A and B) Protein-interacting networks associated with the DEN group. (C and D) Protein-interacting networks associated with the DEN+PDTC group. (E and F) Protein-interacting networks associated with the DEN+LPS group. These networks were centered on HNF4A, actin, GRB2, LMNA, EGFT and ICAM1. The intensity of the color represents the degree of upregulation (red) or downregulation (green) of genes in the dataset. Molecular types represented by each node shape are shown. 
A

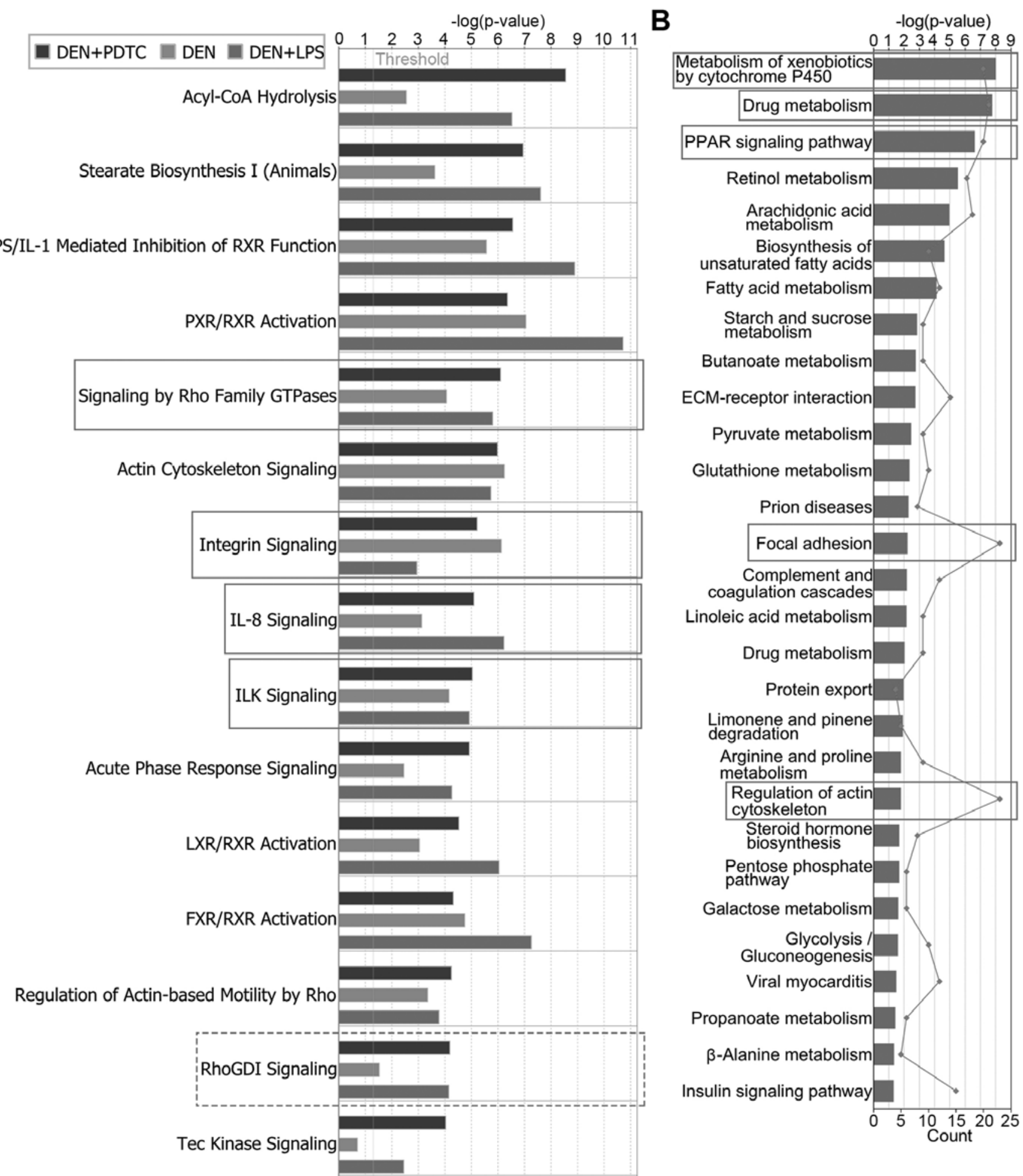

Figure 4. Pathway analyses revealed the changing regulatory pathways associated with EMT. (A) Canonical pathway analysis using IPA. Commonly activated or inhibited signaling pathways among the three treatment groups are labeled with solid boxes (activated) or dotted boxes (inhibited). (B) KEGG enrichment analysis. The main axis with column chart demonstrates -log (p-value). The secondary axis with line chart demonstrates the number of differentially expressed proteins in the corresponding pathway. Terms labeled with boxes indicate the top five signaling pathways with the most differentially expressed proteins.

and DEN+LPS groups. By contrast, RhoGDI signaling was the common inhibited pathway. All these pathways had a close relationship with EMT-related cell adhesion and migration.

To obtain precise results, we further uploaded the differentially expressed proteins into the DAVID database (https:// david.ncifcrf.gov/) for KEGG signaling pathway enrichment analysis. A total of 29 pathways with $\mathrm{p}<0.05$ were enriched (Fig. 4B). By ranking the number (line chart in Fig. 4B) of differentially expressed proteins in each pathway, the top five signaling pathways were focal adhesion (Fig. 5A), regulation of actin cytoskeleton, drug metabolism by cytochrome P450, metabolism of xenobiotics by cytochrome P450, and PPAR 


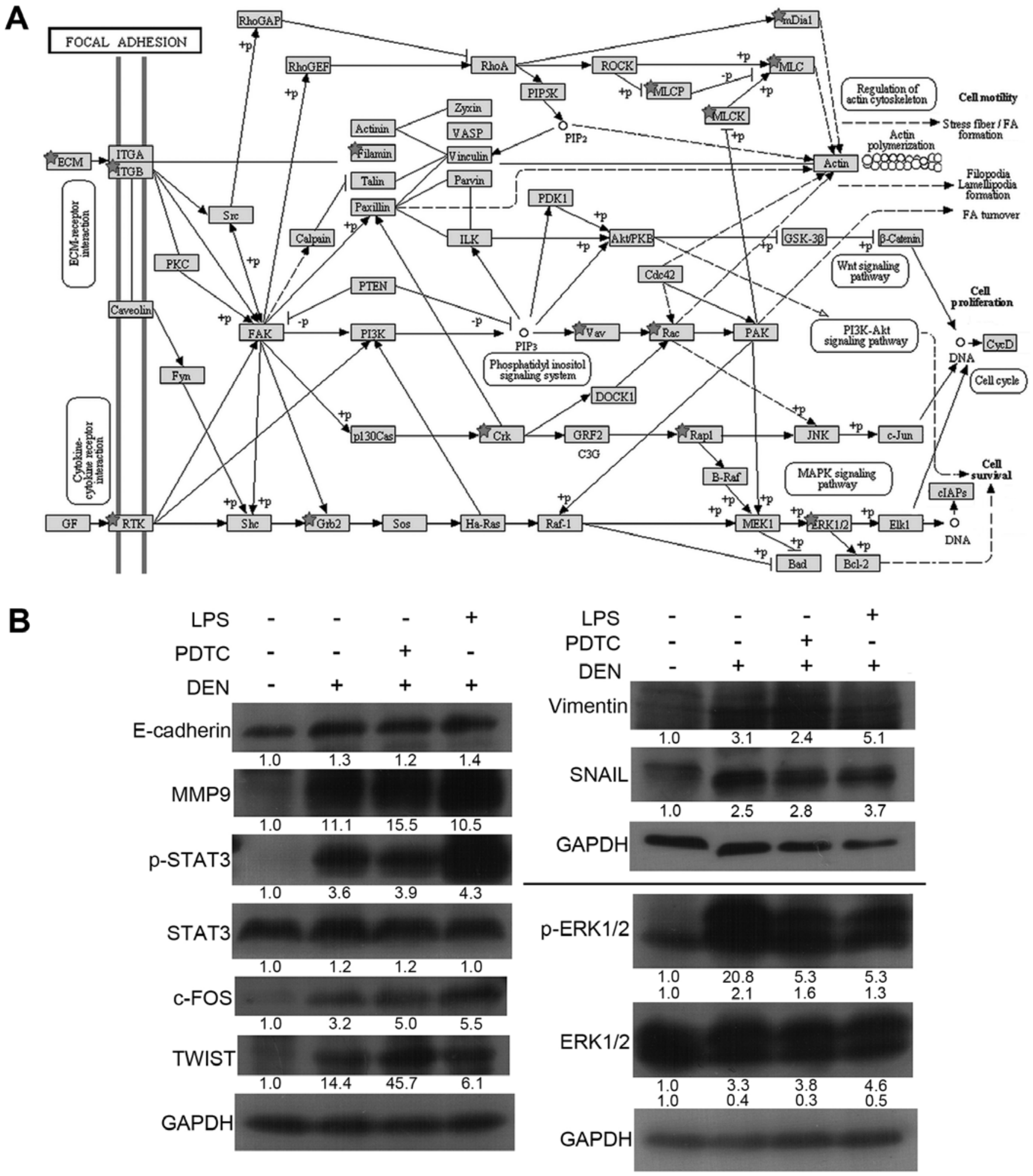

Figure 5. Pathway of focal adhesion from KEGG and western blot analysis. (A) Pathway of focal adhesion. Molecules labeled with stars are differentially expressed proteins mapped to the pathway. (B) Western blot analysis. The number below the band indicate protein levels relative to GAPDH. Band intensity was calculated with Quantity One (version 4.6.2, Bio-Rad) and normalized to control group (the first lane on the left).

signaling pathway (data not shown). Drug metabolism, metabolism of xenobiotics by cytochrome P450, and PPAR signaling pathway are closely related to inflammatory responses and oxidative stress, which is consistent with the results of the IPA tox list analysis. Moreover, focal adhesion and the regulation of actin cytoskeleton are closely associated with cell adhe- sion and migration, which supported the results of the IPA canonical pathway analysis.

To further verify the enhanced EMT ability in hepatic cells from proteomics data, we detected the expression levels of some key factors in EMT by western blot analysis. As shown in Fig. 5B, EMT-associated key proteins, such as 
p-STAT3, TWIST, SNAIL, Vimentin, and MMP-9, were all upregulated in the three treatment groups, which demonstrated that hepatic cells had access to EMT. E-cadherin is a key protein that was expected to be downregulated in EMT. Interestingly, E-cadherin also increased in hepatic cells during the inflammation stage. According to the focal adhesion pathway (Fig. 5A), the ERK signaling pathway was activated as a downstream target of integrin signaling. This trend was confirmed by the increasing levels of phosphorylated ERK1/2 and its downstream transcription factor, c-FOS (Fig. 5B).

\section{Discussion}

HCC is the third leading cause of cancer-related death worldwide (10). The condition often develops in the context of chronic hepatitis, which is mainly caused by viruses or toxic compounds $(11,12)$. In-depth research has provided a clear understanding of the relationship between inflammation and liver cancer. However, the precise underlying mechanism has not been completely elucidated. The study of cellular pathological changes and their regulatory mechanisms during the early inflammatory period will contribute toward accelerating HCC diagnosis and prevention. Having established a mouse inflammation-cancer model successfully, we performed a systemic exploration on the molecular pathogenic mechanisms during the precancerous inflammatory period via LFQ proteomics technology combined with bioinformatics analysis.

Our results demonstrated a common trend of inflammation in the three treatment groups. DEN has been widely recognized to cause liver cell injury and induce hepatocellular carcinoma. The role of LPS in promoting inflammation has also been confirmed. However, PDTC impairs the inflammatory response by inhibiting the synthesis of NOS and the activation of $\mathrm{NF}-\kappa \mathrm{B}$. H\&E staining of mouse liver tissues showed that the infiltration of inflammatory cells had occurred in DEN, DEN+PDTC, and DEN+LPS groups (Fig. 1A). Our final results proved that mice in all three groups had formed hepatic cirrhosis (9). In heat map of HCA, similarities were found among the three treatment groups against the control group. The Venn diagram showed that 204 upregulated proteins and 62 downregulated proteins were shared by the three treatment groups. All the results indicated that in the complex regulatory process, some common regulatory mechanisms allowed the sustainable inflammatory microenvironment to induce the conversion of hepatic cells to the malignant phenotype. We performed cellular component enrichment analysis on the 204 common upregulated proteins and found that these proteins were mainly components of the extracellular matrix and cytoskeleton, which were involved in cell adhesion and cell junctions. This trend was consistent with our subsequent pathway analysis.

Tox list analysis using IPA revealed the association of our data set with oxidative stress-related pathological processes. $\mathrm{RXR}$, also called the retinoid $\mathrm{X}$ receptor, is a member of nuclear receptor superfamily. RXR can repress apoptosis by impairing oxidative stress in cells (13-16). Cytochrome P450, LPS, and NRF2 are also closely related to oxidative stress. In addition, some representative proteins related to liver injury had significantly changed after experiencing sustainable inflam- mation according to our proteomic data. For instance, major urinary protein (MUP) is a protein family mainly expressed in the liver; the expression levels of proteins in this family significantly decreased after a liver injury (17). In our data, the expression levels of MUP2, MUP3, MUP6, and MUP20 were notably decreased. Other liver injury-related proteins, such as lactotransferrin (TRFL) and haptoglobin (HPT) (18), were also significantly changed (data not shown). Therefore, hepatic cells suffered serious oxidative stress-induced injury. Subsequent western blot experiments to detect Caspase-3, BAX, and LC3B demonstrated the death of hepatic cells.

Protein-interacting networks were constructed by IPA and depicted some protein functional groups associated with cellular assembly and organization, DNA replication, recombination, and repair, developmental disorders, hereditary disorders, the cell cycle, lipid metabolism, nucleic acid metabolism, and organismal injury and abnormalities. To further dissect the specific regulatory events, the central node proteins were subject to functional retrieval based on UNIPROT (http:// www.uniprot.org/) and previously published study. As the central node of two networks, hepatocyte nuclear factor 4- $\alpha$ (HNF4A) attracted our attention. Growing evidence suggested that HNF4A plays a role in the inflammatory-cancer loop (19), but the exact molecular mechanisms have yet to be defined. HNF4 is a transcriptional regulation factor that belongs to a family of hormone receptors. The protein is involved in regulating lipid and glucose metabolism, cell junctions, and the differentiation and proliferation of liver and intestinal epithelial cells (20). Zeisberg et al (21) found that HNF4A was downregulated in mouse liver cells during EMT induced by TGF $\beta$. Rygiel et al (22) also proved that the heterogeneous expression of HNF4A could prevent EMT in hepatic cells. Our data demonstrated the downregulation of HNF4A during the inflammation stage. This trend directed our attention to EMT, which was consistent with the pathways analysis.

Canonical pathway analysis by IPA demonstrated some common pathways shared by the DEN, DEN+PDTC, and DEN+LPS groups. Integrin signaling, signaling by Rho family GTPase, IL-8 signaling, and ILK signaling were activated, whereas RhoGDI signaling was inhibited. Integrin was reported to regulate EMT via focal adhesion kinase (FAK) (23) or integrin linked kinase (ILK) (24). The signaling of PI3K/AKT (25), WNT (26), and RAS/MAPK (27) are all downstream targets of integrin signaling. Members of the Rho GTPase family are mainly involved in the regulation of cytoskeletal and cell adhesion (28); RhoGDI is an important negative regulator of Rho GTPase. These results indicated the changes of hepatic cells in terms of adhesion, migration, or EMT. Further KEGG pathway enrichment analysis revealed that the signaling pathways of focal adhesion and the regulation of the actin cytoskeleton were significantly changed. As shown in Fig. 5A, the integrin-mediated signaling played a central role in regulating the cytoskeleton, cell adhesion, and cell migration, which supported the IPA results. The upregulation of p-STAT3, TWIST, SNAIL, Vimentin, and MMP-9 strongly demonstrated the tendency of EMT in hepatic cells. Regardless of the unexpected increase of E-cadherin, the migration of hepatic cells during the inflammation stage was enhanced, and the potential EMT tendency should not be ignored. 
In concusion, this study provides a global view on the transformation of hepatic cells exposed to inflammation. Despite the complexity of regulatory mechanism by which the inflammatory microenvironment transforms hepatic cells and the limitations of bioinformatics analysis, which needs verification by further experiments, our study still partially reveals the underlying mechanism of transformation. Under a sustainable inflammatory microenvironment, hepatic cells suffer serious oxidative stress-induced injury and death. Downregulated HNF4A may be a warning marker for liver injury and further hepatic cell deterioration. The regulatory network centered on integrin signaling increases migration and the EMT ability of hepatic cells by regulating the cytoskeleton and focal adhesion. The involvement of HNF4A in this regulatory network still needs to be defined.

\section{Acknowledgements}

This study was supported by National Natural Science Foundation of China (Grant Nos. 81272921,81272245, 81272445 and 81471970); Joint Programme by Healthy Care System and Educational Department in Fujian Province (Grant No. WKJ-FJ-16); The Natural Science Foundation of Fujian Province of China (Grant No. 2013D004); the Fundamental Research Funds for the Central Universities (Grant No. 20720140545).

\section{References}

1. Karin M: Nuclear factor-kappaB in cancer development and progression. Nature 441: 431-436, 2006.

2. Kuper H, Adami HO and Trichopoulos D: Infections as a major preventable cause of human cancer. J Intern Med 248: 171-183, 2000.

3. Allavena P, Garlanda C, Borrello MG, Sica A and Mantovani A: Pathways connecting inflammation and cancer. Curr Opin Genet Dev 18: 3-10, 2008.

4. Nakagawa $\mathrm{H}$ and Maeda S: Inflammation- and stressrelated signaling pathways in hepatocarcinogenesis. World J Gastroenterol 18: 4071-4081, 2012.

5. Balkwill F and Mantovani A: Inflammation and cancer: Back to Virchow? Lancet 357: 539-545, 2001.

6. Markiewski MM, DeAngelis RA and Lambris JD: Liver inflammation and regeneration: Two distinct biological phenomena or parallel pathophysiologic processes? Mol Immunol 43: 45-56, 2006.

7. Connor JH, Weiser DC, Li S, Hallenbeck JM and Shenolikar S: Growth arrest and DNA damage-inducible protein GADD34 assembles a novel signaling complex containing protein phosphatase 1 and inhibitor 1. Mol Cell Biol 21: 6841-6850, 2001.

8. Poirier MC: Chemical-induced DNA damage and human cancer risk. Discov Med 14: 283-288, 2012.

9. Peng B, Liu F, Han R, Luo G, Cathopoulis T, Lu K, Li X, Yang L, Liu GY, Cai JC, et al: Dynamic metabolic change is indicative of inflammation-induced transformation of hepatic cells. Int J Biochem Cell Biol 66: 45-58, 2015.

10. El-Serag HB and Rudolph KL: Hepatocellular carcinoma: Epidemiology and molecular carcinogenesis. Gastroenterology 132: 2557-2576, 2007.

11. Berasain C, Castillo J, Perugorria MJ, Latasa MU, Prieto J and Avila MA: Inflammation and liver cancer: New molecular links. Ann NY Acad Sci 1155: 206-221, 2009.
12. Nikolaou K, Sarris M and Talianidis I: Molecular pathways: The complex roles of inflammation pathways in the development and treatment of liver cancer. Clin Cancer Res 19: 2810-2816, 2013.

13. Zhao XR, Gonzales N and Aronowski J: Pleiotropic role of PPAR $\gamma$ in intracerebral hemorrhage: An intricate system involving Nrf2, RXR, and NF- $\kappa B$. CNS Neurosci Ther 21: 357-366, 2015.

14. Shan P, Pu J, Yuan A, Shen L, Shen L, Chai D and He B: RXR agonists inhibit oxidative stress-induced apoptosis in $\mathrm{H} 9 \mathrm{c} 2$ rat ventricular cells. Biochem Biophys Res Commun 375: 628-633, 2008.

15. Chai D, Wang B, Shen L, Pu J,Zhang XK and He B: RXR agonists inhibit high-glucose-induced oxidative stress by repressing PKC activity in human endothelial cells. Free Radic Biol Med 44: 1334-1347, 2008.

16. Elbrecht A, Chen Y, Cullinan CA, Hayes N, Leibowitz M, Moller DE and Berger J: Molecular cloning, expression and characterization of human peroxisome proliferator activated receptors gamma 1 and gamma 2. Biochem Biophys Res Commun 224: 431-437, 1996.

17. Elchuri S, Naeemuddin M, Sharpe O, Robinson WH and Huang TT: Identification of biomarkers associated with the development of hepatocellular carcinoma in $\mathrm{CuZn}$ superoxide dismutase deficient mice. Proteomics 7: 2121-2129, 2007.

18. Bell LN, Vuppalanchi R, Watkins PB, Bonkovsky HL, Serrano J, Fontana RJ, Wang M, Rochon J and Chalasani N; US DrugInduced Liver Injury Network (DILIN) Research Group: Serum proteomic profiling in patients with drug-induced liver injury. Aliment Pharmacol Ther 35: 600-612, 2012.

19. Hatziapostolou M, Polytarchou C, Aggelidou E, Drakaki A, Poultsides GA, Jaeger SA, Ogata H, Karin M, Struhl K, Hadzopoulou-Cladaras M, et al: An HNF4a-miRNA inflammatory feedback circuit regulates hepatocellular oncogenesis. Cell 147: 1233-1247, 2011.

20. Babeu JP and Boudreau F: Hepatocyte nuclear factor 4-alpha involvement in liver and intestinal inflammatory networks. World J Gastroenterol 20: 22-30, 2014

21. Zeisberg M, Yang C, Martino M, Duncan MB, Rieder F, Tanjore $\mathrm{H}$ and Kalluri R: Fibroblasts derive from hepatocytes in liver fibrosis via epithelial to mesenchymal transition. J Biol Chem 282: 23337-23347, 2007.

22. Rygiel KA, Robertson H, Marshall HL, Pekalski M, Zhao L, Booth TA, Jones DEJ, Burt AD and Kirby JA: Epithelialmesenchymal transition contributes to portal tract fibrogenesis during human chronic liver disease. Lab Invest 88: 112-123, 2008.

23. Figel S and Gelman IH: Focal adhesion kinase controls prostate cancer progression via intrinsic kinase and scaffolding functions. Anticancer Agents Med Chem 11: 607-616, 2011.

24. Cortez V, Nair BC, Chakravarty D and Vadlamudi RK: Integrinlinked kinase 1: Role in hormonal cancer progression. Front Biosci (Schol Ed) 3: 788-796, 2011.

25. Ke AW, Shi GM, Zhou J, Huang XY, Shi YH, Ding ZB, Wang XY, Devbhandari RP and Fan J: CD151 amplifies signaling by integrin $\alpha 6 \beta 1$ to PI3K and induces the epithelial-mesenchymal transition in HCC cells. Gastroenterology 140: 1629-41.e15, 2011.

26. Gil D, Ciołczyk-Wierzbicka D, Dulińska-Litewka J, Zwawa K, McCubrey JA and Laidler P: The mechanism of contribution of integrin linked kinase (ILK) to epithelial-mesenchymal transition (EMT). Adv Enzyme Regul 51: 195-207, 2011.

27. Hwangbo C, Kim J, Lee JJ and Lee JH: Activation of the integrin effector kinase focal adhesion kinase in cancer cells is regulated by crosstalk between protein kinase Calpha and the PDZ adapter protein mda-9/Syntenin. Cancer Res 70: 1645-1655, 2010.

28. Fuse T, Kanai Y, Kanai-Azuma M, Suzuki M, Nakamura K, Mori H, Hayashi Y and Mishina M: Conditional activation of RhoA suppresses the epithelial to mesenchymal transition at the primitive streak during mouse gastrulation. Biochem Biophys Res Commun 318: 665-672, 2004. 